\title{
Displacements in a Column-Reinforced Granular Medium: A Probabilistic Approach
}

\author{
Djamalddine Boumezerane ${ }^{1}$, Meryem Lahbiben ${ }^{2}$ \\ ${ }^{1}$ Geotechnical Division, Norwegian University of Science and Technology - NTNU \\ Gløshaugen 7491, Trondheim, Norway \\ Djamalddine.boumezerane@ntnu.no \\ ${ }^{2}$ Civil Engineering Department, University of Bejaia - UAMB \\ Route Targa Ouzemmour 06000, Bejaia, Algeria
}

\begin{abstract}
A probabilistic approach was proposed by Harr [1] to account for diffusion of stresses in a granular medium. It considers that a point load applied on the surface of a granular media will follow an erratic path, depending on the probability of transition between the grains. The diffusion of the expected vertical stress in the granular medium obeys a similar equation as the known FokkerPlanck equation. Bourdeau [2] extended this approach to the diffusion of vertical displacements in a cohesionless granular medium. An equation of diffusion is obtained and the parameter of diffusion is shown to approximate the mean diameter of the material grains. We revisited the formulation of displacements diffusion to account for a gravel column-reinforced soil submitted to foundation loading. A numerical approach based on finite elements method is used to account for a gravel column-reinforced soil submitted to loadings of a building foundation. The obtained results show that the diffusion of displacements in the granular medium is influenced by the grain size distribution and it helps using the method as a valuable tool to calculate settlements. Comparisons are shown when the soil (granular medium) is reinforced with gravel columns.
\end{abstract}

Keywords: Granular media, Diffusion, Displacements, Gravel column, Probability

\section{Introduction}

Soils are constituted of an assembly of particles of different sizes, mineralogy and morphologies. The particle sizes vary from less than $0.002 \mathrm{~mm}$ in clays to some tens of millimeters in gravel materials. Despite their particular (granular) aspect, they are considered, from the soil mechanics viewpoint, as a continuum and elastoplastic theories are applied to model the behavior of such materials. Critical state approach is one of them and it models well the behavior of clayey soils which is mostly driven by volumetric deformations. Cohesionless soils behavior on the other hand is difficult to capture using elastoplacity approaches. The granular aspect of these soils, especially when they are in loose states, makes their behavior complex to predict using elastoplastic theory.

Harr [1] proposed an approach based on probability theory to study the behavior of granular materials subjected to static loads. The main idea of this approach is that a point load applied on the surface of a particulate media will follow a random path given a probability law. An equation of diffusion is obtained, expressed in terms of expected intergranular stresses and depending on the lateral pressure coefficient. Bourdeau [2] extended the concept to study settlements in cohensionless loose soils. The author investigated the response of loose cohesionless soils to surface applied loads. The proposed model is based on the combination of the diffusion of intergranular forces and an excess volume of voids. The model was applied in the case of homogenous particulate media.

In this work an attempt is made within the same approach to simulate the response of gravel column-reinforced soil under loading of a building foundation. We first revisit the diffusion equation in terms of displacements, and use a finite element code to simulate two layers of cohesionless soil submitted to a foundation loading. Gravel column reinforcement is introduced and a comparative study is made with available results. 


\section{Principles}

A concentrated load applied on the surface of a semi-infinite ground will follow an uncertain path between the grains. The resulting stress in one point is a random variable. Its distribution will reflect the composition of the media. Following a binomial law of diffusion on the right or on the left of a given reference mark, the load in question is translated in the granular medium as equation of diffusion of stresses.

A formulation in terms of displacements is also possible while following the same reasoning. The media is characterized by a coefficient $\mathrm{D}$ of diffusion and a coefficient $\mathrm{C}$ of convection. The coefficient $\mathrm{D}$ depends on several factors notably the mean diameter of the grains [2].

\section{Probabilistic model}

The application of a vertical load on a surface of a granular media randomly arranged, and statistically homogeneous, can be considered as the sum of a number of elementary, discreet loads. By neglecting the compressibility of the grains and their deformation, the resulting inter-granular stress of a normal elementary load at the surface will follow an erratic path through contacts between grains of the media (soil), without producing deformation until it reaches an unsteady assembly. In addition, the consequence of this movement will produce an elementary deflection in the surface, the resulting deflection being proportional to the cumulative voids provoked in the granular media [3].

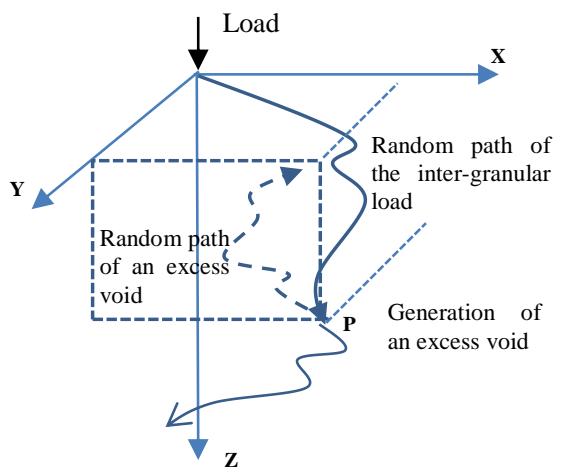

Fig. 1: Generation of excess void in a granular medium (after [3]).

The phenomenological model of the loading on the surface of a granular media is constituted of three components (Fig.1). The first is the propagation of the intergranular load, the second, is the generation of excessive voids and the third component is a consequence of these. It consists of a migration upwards of the voids.

The second component is the tie between the first and the third steps (phenomenon of propagation)

Let $A(p)$ be the event "Force crossing the point $\mathrm{p}$ at $\mathrm{z}$ depth" and $B(p)$ be the event "Creation of a void at the same point".

The probability of the occurrence of the two events A and B is:

$$
V=\operatorname{Prob}(A \cdot B)=\operatorname{Prob}(B \mid A) \operatorname{Prob}(A)
$$

With $\operatorname{Prob}(B \mid A)$ a conditional probability.

Let us consider an elementary volume $\Delta V$ of dimension $\Delta X . \Delta Y . \Delta Z$ around , we have:

$$
V=\operatorname{Prob}(A . B) \Delta X . \Delta Y . \Delta Z
$$

Therefore, the joined probability $\operatorname{Prob}(A . B)$ is equivalent to the volumetric distortion of the element of volume $\Delta V$. The conditional probability $\operatorname{Prob}(B \mid A)$ represents the change of volume by unit of stress [4]. 


\subsection{Formulation of the diffusion equation}

A parallel was made between Brownian motion of particles in sedimentation in a viscous fluid and the random diffusion of forces between the grains of a medium [2].

In the theory of the Brownian motion, the differential equation of diffusion of particles in a fluid according to their concentration is given by

$$
\frac{\partial f}{\partial t}=D_{z} \frac{\partial^{2} f}{\partial z^{2}}-C_{z} \frac{\partial f}{\partial z}
$$

$f(t)$ : Concentration of the particles depositing in a viscous fluid that varies during the time;

$C_{z}$ and $D_{z}$ Coefficients that depend on the fluid.

Given the analogy between volumetric concentration of the particles depositing at $\mathrm{z}$ depth of a liquid and the probability of the presence of an isolated particle in one interval of the space, equation (3) can be written in terms of probabilities as

$$
\frac{\partial f_{z}(z, t)}{\partial t}=D_{z} \frac{\partial^{2} f_{z}(z, t)}{\partial z^{2}}-C_{z} \frac{\partial f_{z}(z, t)}{\partial z}
$$

Where $f_{z}$ is the density of probability of transition from the state $\mathrm{z} 0$ to the state $\mathrm{z}$ in an interval ( $\mathrm{t}-\mathrm{t} 0$ ).

The passage, from equation (4) of diffusion of particles in a fluid toward an equation in the same type in terms of displacements in a granular media, is obtained when replacing the variable $(\mathrm{t})$ of time with a variable $(\mathrm{z})$ of depth [4].

$$
\frac{\partial f(x, z)}{\partial z}=\frac{\partial^{2}[D \cdot f(x, z)]}{\partial x^{2}}-\frac{\partial[C \cdot f(x, z)]}{\partial x}
$$

The equation can be written in terms of displacements in XOZ plane

$$
\frac{\partial W(x, z)}{\partial z}=D \cdot \frac{\partial^{2} W(x, z)}{\partial x^{2}}-C \cdot \frac{\partial W(x, z)}{\partial x}
$$

In this equation, the mean displacement $W$ plays the role of the concentration by unit of surface of the distributed substance.

In a ZOY plane, it will be expressed as:

$$
\frac{\partial W(y, z)}{\partial z}=D \cdot \frac{\partial^{2} W(y, z)}{\partial y^{2}}-C \cdot \frac{\partial W(y, z)}{\partial y}
$$

In cylindrical coordinates, by combining (6) and (7) we can write

$$
\frac{\partial W}{\partial z}=D \cdot\left(\frac{1}{r} \cdot \frac{\partial W}{\partial r}+\frac{\partial^{2} W}{\partial r^{2}}\right)-\frac{\partial}{r}(C . W)
$$

Where:

$D$ is the Coefficient of diffusion.

$C$ is the Coefficient of convection (in our case $C=0$, only the diffusion is major in the granular medium).

$W:$ is the vertical displacement 
Equation (6) is rewritten as;

$$
\begin{gathered}
-<\frac{\partial W}{\partial x} \frac{\partial W}{\partial z}>\left(D\left\{\begin{array}{c}
\frac{\partial W}{\partial x} \\
\frac{\partial W}{\partial z}
\end{array}\right\}\right)+<C \quad 1>\left\{\begin{array}{c}
\frac{\partial W}{\partial x} \\
\frac{\partial W}{\partial x}
\end{array}\right\}=F(W) \\
d_{a} \frac{\partial W}{\partial t}-\nabla \cdot(D \nabla W)+\beta \cdot \nabla W=F
\end{gathered}
$$

Such

$D$ : Constant

$$
d_{a}=0 ; \quad \beta_{x}=C ; \quad \beta_{z}=1 ; \quad F=-D \frac{\partial^{2} W}{\partial z^{2}}
$$

\subsection{Numerical approach}

Finite element method is used to solve the diffusion equation. Fig.2 shows the boundary conditions. A rigid and uniform loading on the surface provokes a uniform displacement of the loaded area ( $W$ constant). The lower boundary is sufficiently distant to consider the displacements equal to zero $(W=0)$. The lateral boundary of the domain is sufficiently far to consider the variation of $W$ zero, because of the bell distribution of the solution.

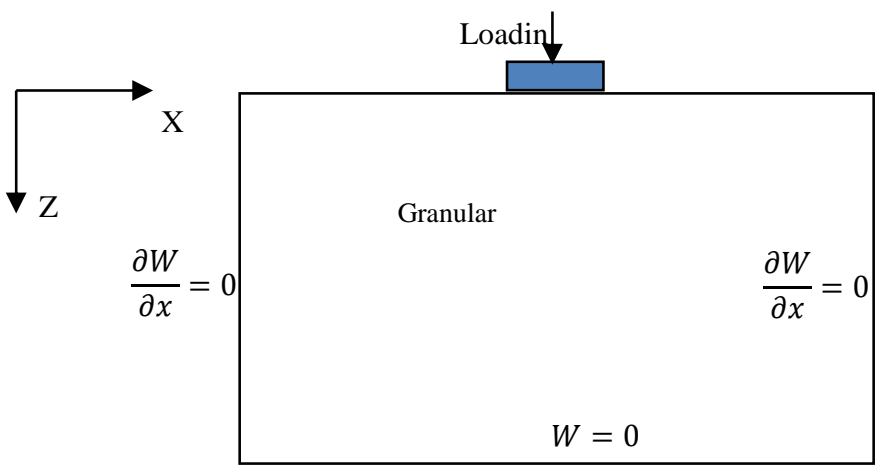

Fig. 2: Boundary conditions of the problem.

The calibration model incorporated in the finite element code COMSOL [5] is as represented in the previous figure. The breadth $\mathrm{B}$ of the plate is $1.20 \mathrm{~m}$, the mean diameter of the grains $\mathrm{D}=2 \mathrm{~mm}$, which considers the medium as sand. Each of the lateral boundaries is $5 \mathrm{~m}$ far from the vertical load axis, and the considered depth is $4 \mathrm{~m}$. The applied pressure is $83 \mathrm{kPa}$.

\section{Parametric study}

\subsection{Influence of grain diameter}

The granular medium is characterized in this study by $D$ the diffusion coefficient, which has a relation with the grain diameter [4]. The same author, Bourdeau [2] gives for different types of soils intervals of values for $D$ in $\mathrm{mm}$

Table1. Grain diameter (mm) for different media (after [2]).

\begin{tabular}{|c|l|l|l|}
\hline Clay & Silt & Sand & Gravel \\
\hline$<0.002 \mathrm{~mm}$ & {$[0.002,0.06 \mathrm{~mm}]$} & {$[0.06,2 \mathrm{~mm}]$} & $>2 \mathrm{~mm}$ \\
\hline
\end{tabular}




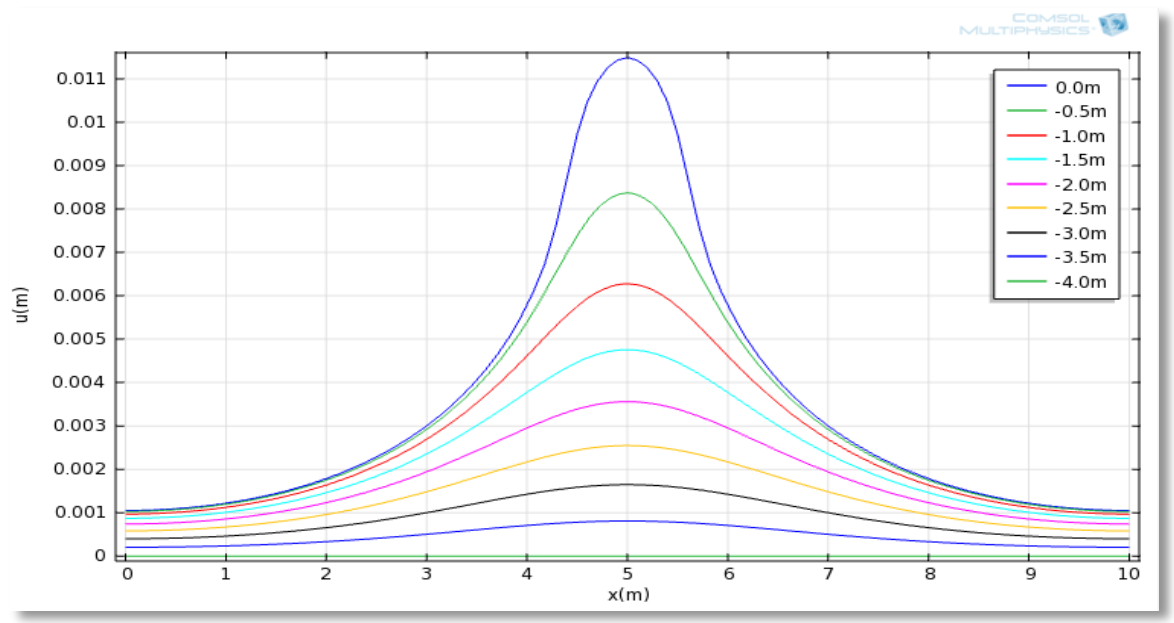

Fig. 3: Diffusion of displacements at different depths.

The validation of the model was performed by comparison of the results with those obtained from the theoretical approach [2]. Fig.4 summarizes the settlements in sand versus depth. A comparison is shown between the present approach, Mohr-Coulomb, Linear elastic models of the finite element code PLAXIS [6] and experimental data from [2].

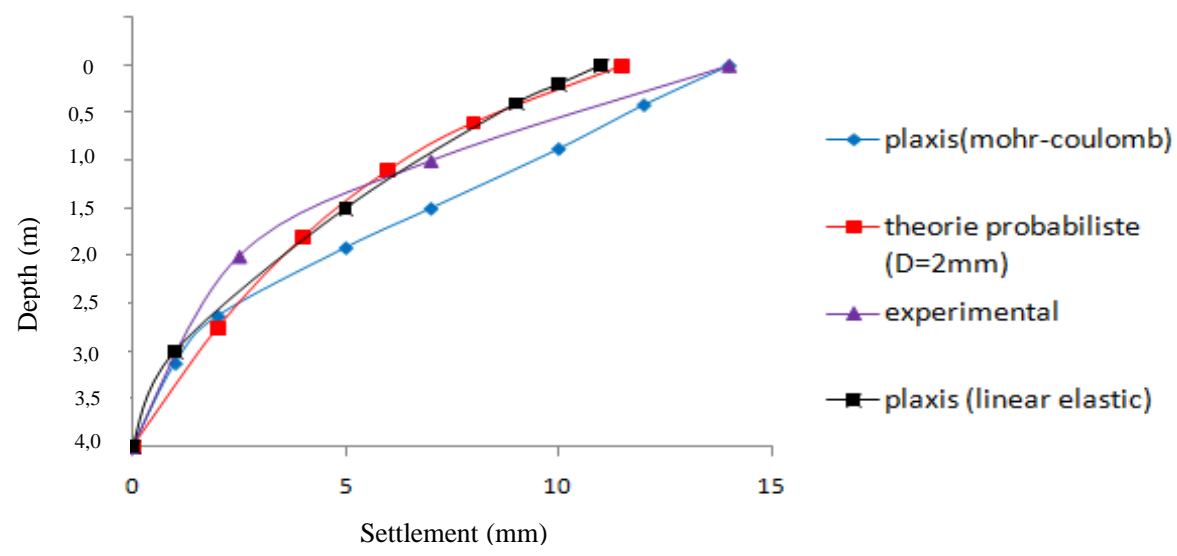

Fig. 4: Settlements in sand vs depth. Comparison with Linear elastic, Mohr-Coulomb and experimental results (after [2]).

\subsection{Case study}

The case study is taken from a project of a building on sandy soil in the region of Sidi Aich (Algeria) [7]. The soil profile is constituted of two main layers of sandy soil as shown in Fig.5. The soil is homogenous composed of coarse sand at the near surface layer of 8.5 meters depth and finer sands over 15 meters below the first layer.

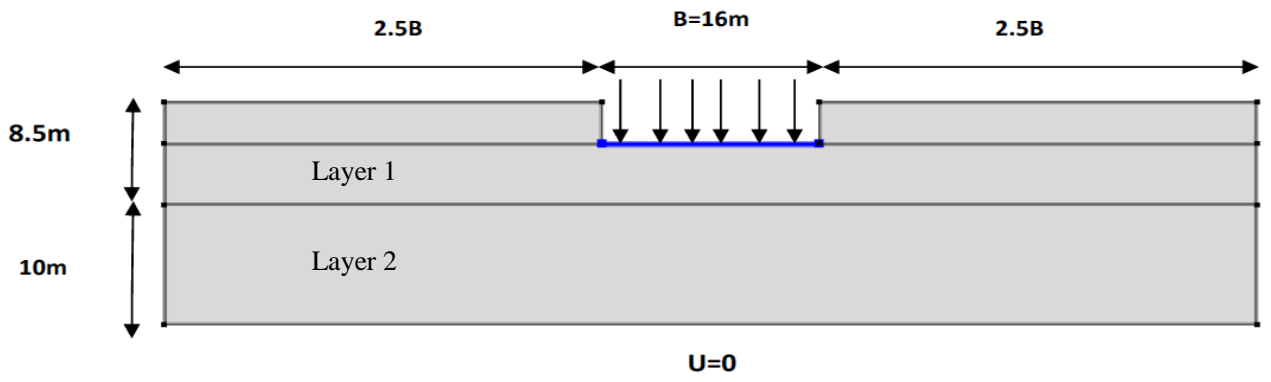

Fig. 5: Profile of two layers of sand. 
Fig.6 shows the curves of displacements at different depths for fixed diffusion parameters, $\mathrm{D}_{1}$ for first layer and $\mathrm{D}_{2}$ for the second layer. The process of diffusion is well illustrated in this case. Table 2 summarises the results when one parameter is kept constant while the other is varying and vice-versa.

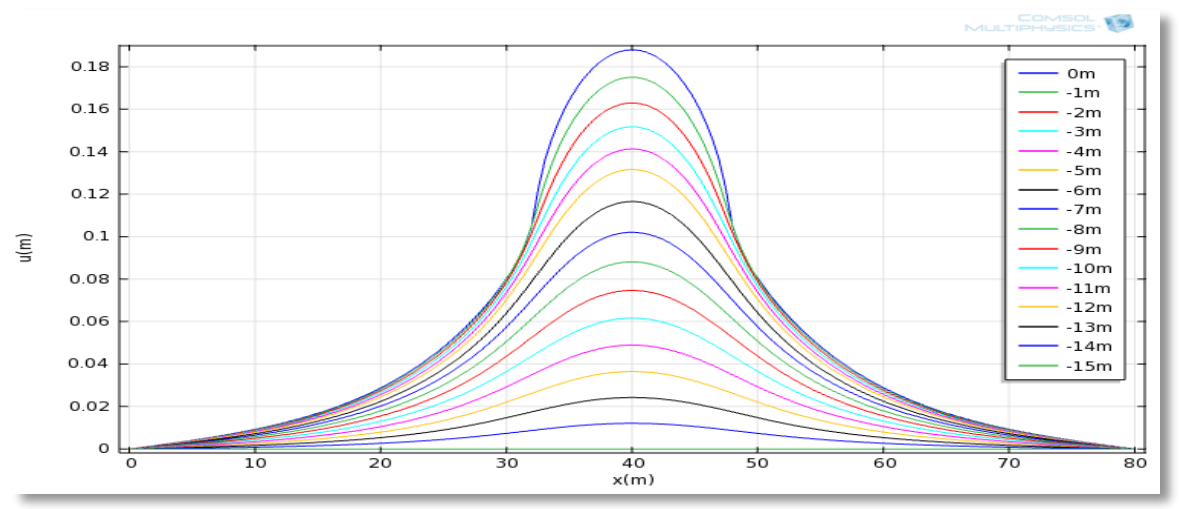

Fig. 6: Curves of displacements diffusion at different depths $\left(\mathrm{D}_{1}=1 \mathrm{~mm}, \mathrm{D}_{2}=0.6 \mathrm{~mm}\right)$.

Table 2. Settlements under foundation axis for different D.

\begin{tabular}{|l|l|l|}
\hline $\begin{array}{l}\text { D1 }(\mathrm{mm}) \\
\text { Upper layer }\end{array}$ & $\begin{array}{l}\text { D2 }(\mathrm{mm}) \\
\text { Lower layer }\end{array}$ & Settlement $(\mathrm{cm})$ \\
\hline 1 & 0.6 & 19 \\
\hline 1.5 & 0.6 & 23 \\
\hline 2 & 0.6 & 27 \\
\hline 1 & 1 & 14.5 \\
\hline 1 & 1.5 & 12 \\
\hline 1 & 2 & 10.5 \\
\hline
\end{tabular}

$\mathrm{D}_{1}$ and $\mathrm{D}_{2}$ denote the mean diameter of the material of layer 1 and layer 2 respectively. It is shown from the above results that when $D_{2}$ is fixed and $D_{1}$ is varying the settlements vary the same way as $D_{1}$, i.e. the higher $D_{1}$ the higher the settlements. On the other hand fixing $\mathrm{D}_{1}$ and varying $\mathrm{D}_{2}$ of the lower layer one can notice that the settlements decrease when $\mathrm{D}_{2}$ increases. The first situation is contradictory as the settlement increases with an increasing $\mathrm{D}_{1}$, but from the second situation a varying $\mathrm{D}_{2}$ when $\mathrm{D}_{1}$ is fixed, implies that the behavior of the system is governed by the compressibility of the lower layer $\left(\mathrm{D}_{2}\right)$. The upper layer of material diameter $\mathrm{D}_{1}$ plays a role of diffusion for displacements.

\subsection{Analysis of a column-reinforced soil}

The geometric conditions are the same as in the first case and gravel columns of diameter $0.8 \mathrm{~m}$ are introduced as a reinforcement. Fig. 5 shows the arrangement of the columns under the foundation area. Grain size distribution of the columns gravel material is in the range $15 \mathrm{~mm}$ to $25 \mathrm{~mm}$ [7]. The model is run using gravel with mean grain diameter $\mathrm{D}=20 \mathrm{~mm}$.

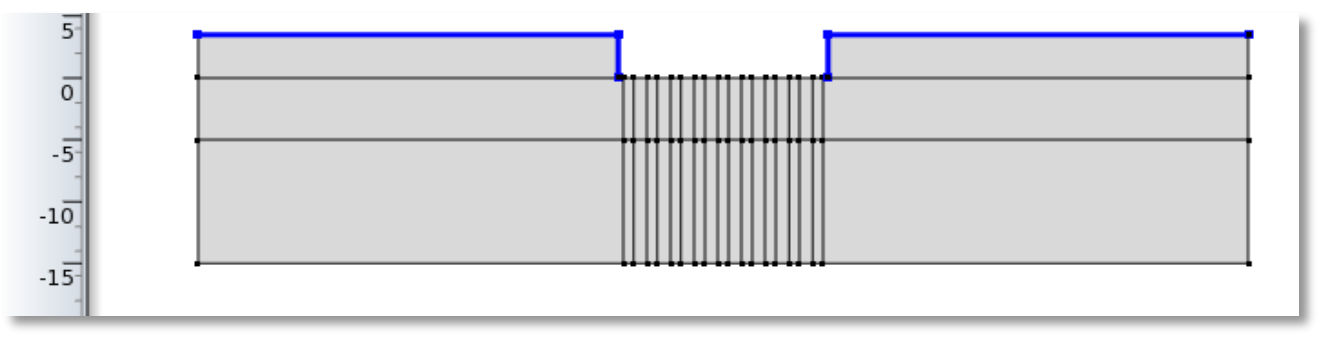

Fig. 5: Soil mass with reinforcing columns. 


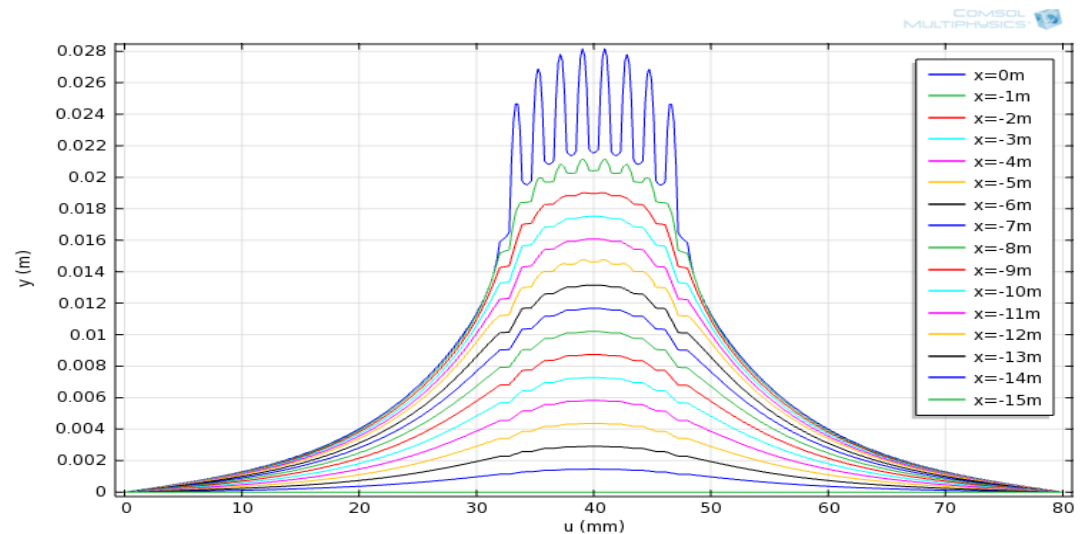

Fig. 6: Diffusion of displacements in the column-reinforced soil mass.

We notice that gravel column-reinforcement reduced drastically the displacements in the whole medium. A study on optimizing the space between columns could be performed, but due to limitation of space in this article, we are limited to this configuration.

We performed a parametric study to examine model sensitivity to the principal parameters (Load and mean diameter of column's gravel). The comparison criteria is the settlement at the surface of the center of the loaded area.

Important variations of settlements due to load change are noticed as expected. In addition, the displacements at the surface are inversely proportional to the mean diameter of the grains. When grains diameter increases the displacements decreases as observed in reality. In sand we encounter less voids and the contact between finer grains are much more numerous than in grosser grains, which induces more transmission of energy and higher displacements at the surface. On the other side in gravels, fewer contacts exist and much more effort is needed for rearrangement of the grains, which implies fewer displacements at the surface. Reinforcement of the soil mass with gravel columns reduced significantly the settlements.

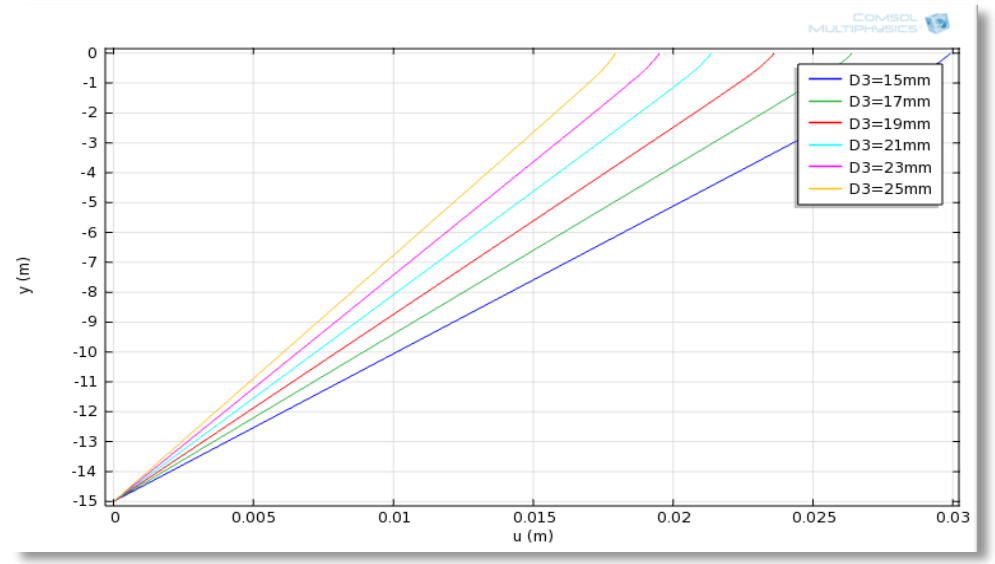

Fig. 7: Influence of the diameter of reinforcement gravel on settlements. 


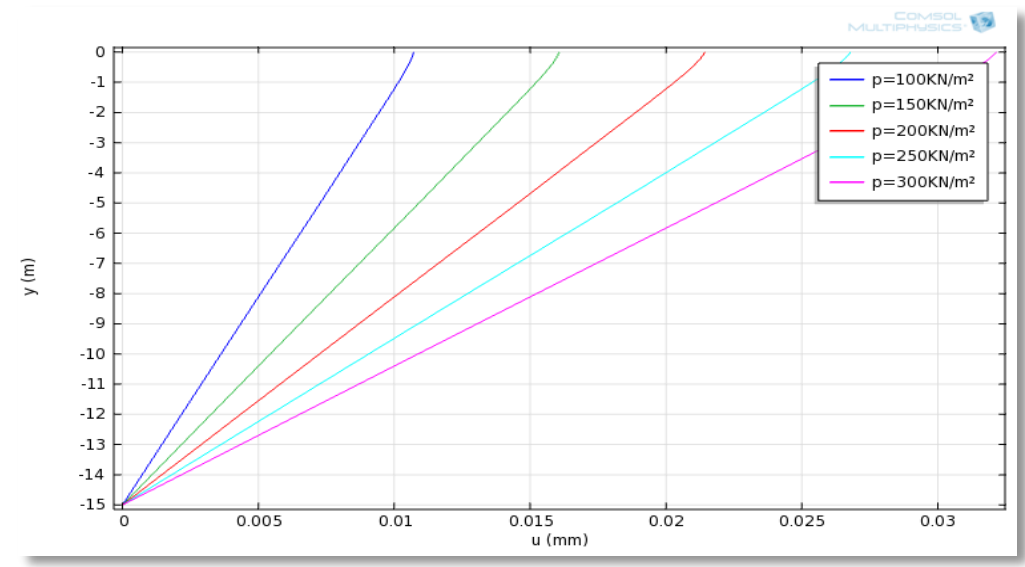

Fig. 8: Influence of the load on settlements.

\section{Conclusion}

A practical approach, based on intergranular forces diffusion in a granular medium such as sand type soils, is revisited to account for soil reinforcement. The gravel column-based reinforcement is treated as part of the granular media with a different parameter of diffusion. The diffusion coefficient is taken as the mean diameter of the grains constituting the medium. The calibration of the model and the obtained results suggest that this approach is valuable in dry cohesionless soils, and it can be used to predict settlements.

\section{References}

[1] M. E. Harr, Mechanics of Particulate Media, a Probabilistic Approach. McGraw-Hill, 1977.

[2] P. L. Bourdeau, "Analyse probabiliste des tassements d'un massif de sol granulaire," Ph.D. dissertation, Dept. Civil Eng. Lausanne Polytechnic, Switzerland, 1986.

[3] P. L. Bourdeau \& M.E. Harr, "Stochastic theory of settlement of loose cohesionless soils," Géotechnique, vol. 39, no. 4, pp. 641-654, 1989.

[4] P. L. Bourdeau, "Stochastic Model of Transient Compression in Granular Media," Powders \& Grains, vol. 93, pp. 429-434, 1993.

[5] Introduction to COMSOL Multiphysics, VERSION 4.2a, 2011.

[6] PLAXIS Version 8. User manual, 2014.

[7] M. Lahbiben, "Tassement d'un milieu granulaire (sol) selon une approche probabiliste," M.S. Thesis, Dept., Civil Eng., University of Bejaia, Algeria, 2014. 